\title{
Microglia in Central Nervous System Diseases
}

\author{
Gary E. Landreth
}

Received: 21 August 2009 / Accepted: 26 August 2009/Published online: 19 September 2009

(C) Springer Science + Business Media, LLC 2009

This special issue of the Journal of Neuroimmune Pharmacology comprises a compilation of papers focused on the roles of the innate immune system in the brain. Microglia are the immune system's representative in the brain and function as the brain's resident macrophage. While it was well know that these cells have diverse functions, a number of new findings have revealed an even richer range of activities and functional states. I think it is fair to say that the biology of microglia and neuroimmunology is now undergoing a renaissance that has arisen as a result of a new understanding of the biology of these cells. This volume highlights the importance of microglial biology and neuroinflammation in a diverse range of diseases of the CNS and infections of the brain and identifies potential approaches for therapeutic intervention in these maladies.

It is rather remarkable that at this late date the natural history of microglia in the brain remains incompletely understood. Dr. Streit highlights a number of these controversies. For example, it is not known with certainty which populations of myeloid lineage cells immigrate into the brain during development and become microglia (Streit and Xue 2009). The average lifespan of a microglia is unknown. Indeed, what is the fate of an aged microglia? Dr. Streit documents a previously unrecognized senescencerelated phenomenon in the human brain, termed cytorrhexis, where microglia become dystrophic, fragment, and die. $\mathrm{He}$

Electronic supplementary material The online version of this article (doi:10.1007/s11481-009-9173-3) contains supplementary material, which is available to authorized users.

G. E. Landreth $(\bowtie)$

Alzheimer Research Laboratory, Department of Neurosciences, Case Western Reserve University School of Medicine,

10900 Euclid Ave.,

Cleveland, OH 44106, USA

e-mail: gel2@case.edu posits that this process may underlie age-related degenerative diseases like Alzheimer's.

Newly developed imaging techniques that allow visualization of microglia in the living brain have revealed new and unexpected activities of these cells. We now know that microglia continuously extend and retract their processes, continuously surveying the entire volume of the brain every few hours. Importantly, these techniques have allowed a clearer understanding of the chronology of pathologic changes in the brain, as discussed by Dr. Combs with respect to Alzheimer's disease (AD) (Combs 2009). These studies have demonstrated the rapid and spontaneous generation of amyloid plaques, the migration of microglia to associate with these deposits and the subsequent dystrophy of adjacent neurites in the brains of animal models of AD. Similarly, the application of PET imaging to evaluation of microglia activation in human disease has been made possible through use of novel ligands which detect activated, but not quiescent microglia. These new findings are also discussed by Dr. Combs with respect AD (Combs 2009) and by Dr. Henkel and colleagues in ALS (Henkel et al. 2009). The development of better PET ligands is underway, suggesting that noninvasive imaging will allow a more thorough understanding of the behavior of these cells in the diseased brain.

While it has been recognized that in diseases of the CNS, like multiple sclerosis, where there is extensive migration of immune cells into the brain, this issue has taken on new importance in the recent past with reports that analogous phenomenon occur in other disorders. This issue has provoked an ongoing controversy on whether peripheral monocytes traffic into the brain in Alzheimer's disease and other neurodegenerative diseases such as Parkinson's. This latter issue is addressed in the papers by Dr. Rezai-Zadeh and colleagues (Rezai-Zadeh et al. 2009) and by Dr. Colton (Colton 2009). 
Historically, microglia have been characterized as either 'activated' or 'quiescent'. It is quite clear that microglia, like their macrophage counterparts, exhibit a broad spectrum of functional states in the diseased brain. Alzheimer's and other degenerative diseases are chronic disorders in which there is a sustained inflammatory response. It has only recently been appreciated that there is substantial phenotypic diversity in the microglia population, with both classically activated microglia and microglia that exhibit quite different characteristics present in the same regions of the diseased brain. These 'alternatively activated' microglia are distinct from either of the former classifications and exhibit some 'anti-inflammatory' properties. These latter characteristics have raised the possibility that the conversion of the microglia into alternative activation states may be of therapeutic utility. This complex biology is the subject of Dr. Colton's contribution (Colton 2009).

This volume summarizes the role of neuroinflammation and the biology of microglia in three common neurodegenerative diseases. Dr. Combs has reviewed the extensive literature on this subject in AD (Combs 2009). It has only relatively recently been appreciated that there is a functionally significant role for microglial-mediated inflammation in Parkinson's Disease (Lee et al. 2009) and in amyotrophic lateral sclerosis (Henkel et al. 2009) and these areas have been reviewed by Dr. Lee and colleagues and Henkel et al., respectively.

Viral and bacterial infection of the brain elicits quite different effects. Dr. Collman discusses the sequelae of HIV infection of the brain and the central role microglia and moncytes/macrophages play in pathology of the brain and cognitive dysfunction (Yadav and Collman 2009). Bacteria and other infectious agents are associated with meningitis, a number of encephalopathies and cerebral malaria. Drs. Mariani and Kielian (2009) describe how these pathogens elicit a microglia response in the brain and the host-defense mechanisms that are engaged to eliminate the pathogens. Dr. Town discusses new data showing that West Nile virus infection of the brain elicits the movement of $\mathrm{T}$ lymphocytes and macrophages across the blood brain barrier (Rezai-Zadeh et al. 2009).

Acknowledgements Work in the Landreth laboratory is supported by grants from the NIA, the Alzheimer's Association, and the Blanchette Hooker Rockefeller Foundation. I would like to thank Ms. Robin Taylor, Managing Editor, and Dr. Howard Gendelman, Editor-in-Chief of the Journal for their assistance in preparing this volume.

\section{References}

Colton CA (2009) Microglial heterogeneity: alternative activation and the innate immune response in the brain. J Neuroimmune Pharmacol (in this issue)

Combs CK (2009) Microglia and Alzheimer's disease. J Neuroimmune Pharmacol (in this issue)

Henkel JS, Beers DR, Zhao W, Appel SH (2009) Microglia in ALS: the good, the bad, and the resting. J Neuroimmune Pharmacol (in this issue)

Lee JK, Tran T, Tansey M (2009) Microglia and neuroinflammation in Parkinson's disease. J Neuroimmune Pharmacol (in this issue)

Mariani M, Kielian T (2009) Microglia in infectious disease of the central nervous system. J Neuroimmune Pharmacol (in this issue)

Rezai-Zadeh K, Gate D, Town T (2009) CNS infiltratin of peripheral immune cells: D-Day for neurodegenerative disease. J Neuroimmune Pharmacol (in this issue)

Streit WJ, Xue QS (2009) Live and death of microglia. J Neuroimmune Pharmacol (in this issue)

Yadav A, Collman R (2009) CNS inflammation and macrophgamicroglial biology associated with HIV-1 infection. J Neuroimmune Pharmacol (in this issue) 